\title{
Mathematical models of non-sinusoidal power supply of a three-phase transverse field MHD inductor
}

\author{
Aleksey Tyapin ${ }^{1}$, Vasiliy Panteleev ${ }^{1}$, and Evgeny Kinev ${ }^{2, *}$ \\ ${ }^{1}$ Siberian Federal University, 79 Svobodny Ave., Krasnoyarsk, 660041, Russia \\ ${ }^{2}$ Thermal Electrical Systems LLC, 12 Spandaryan St., Krasnoyarsk, 660020, Russia
}

\begin{abstract}
The article presents an approach to the development of mathematical models of non-sinusoidal and dual-frequency power supply for a linear induction MHD machine for metallurgical purposes. The issues of construction and numerical modeling of the modes of a three-phase inductor for a liquid aluminum stirrer are considered. Reduction of losses is ensured by the use of a toothless design of the MHD inductor. The absence of steel teeth reduces saturation of the magnetic circuit and current distortion. It is proposed to use the parametric model of the inductor under the furnace in the ANSYS environment to clarify the modes of the complex. To take into account mutual induction, using controlled sources, a circuit model was built, and a numerical calculation of the modes was carried out. The characteristics of instantaneous currents and voltages are obtained when powered from a three-phase source with close frequencies, with pronounced beats. It is shown that the presence of mutual inductance redistributes currents in the delta windings, which must be taken into account when developing the design of linear induction machines. It is proposed to use sources with non-sinusoidal periodic currents in the modeling system. The analysis is carried out and the main types of modulated voltage characteristics in the power supply system of the induction MHD stirrer are shown.
\end{abstract}

\section{Introduction}

Power supply of linear induction machines (LIM) for MHD stirrers of aluminum melt in mixers and furnaces is traditionally performed at a low frequency using frequency converters [1]. At high temperatures and in an aggressive chemical environment, the operating conditions of the complexes are not very simple. The operating modes of three-phase or twophase inverters are characterized by currents of 300-500 amperes, and voltages up to 400 volts. The natural power factor LIM usually does not exceed 0.1 . Therefore, with small losses, the total power of the induction complex can be 500-600 kVA. Stirring of the melt is performed periodically, in cycles of 10-20 minutes during melting, lasting up to several hours. In this cycle, the IGBT transistor converter goes through the stages of start-up, acceleration, reversal, deceleration and shutdown [2]. Considering the high degree of

\footnotetext{
*Corresponding author: Kinev_ES@ontecom.com
} 
unbalance of the inductive load, its static nature and non-sinusoidal consumption currents, the nominal operating mode of the inverter with LIM is essentially emergency for a standard converter designed for a rotating electric drive. Therefore, for metallurgical complexes, specially designed power supplies are used that meet stringent operational requirements [3]. To operate at the edge of the frequency range, at a current frequency of about $1 \mathrm{~Hz}$, inverter modifications are used, based on IGBT modules with limiting operating parameters of 1.2$1.5 \mathrm{kV}$ and $1 \mathrm{kA}$. Nevertheless, it is necessary to apply special protection measures that differ from the standard ones in order to limit overvoltages and extreme currents, especially in emergency power shedding modes [4].

The designs of the windings of the inductors of linear MHD machines are already well studied and worked out [5]. Therefore, the study of nutritional methods for complex solutions is aimed at improving power supplies. One of the search directions can be considered the use of non-sinusoidal currents in the windings [6,7], as well as two-frequency polyphase power supply. Such modes increase the traction efficiency of traditional transverse field inductors [8]. However, each new solution requires careful verification, calculations and modeling, and then a physical experiment.

An example of a melting furnace for recycling aluminum alloys is shown in Fig. 1.

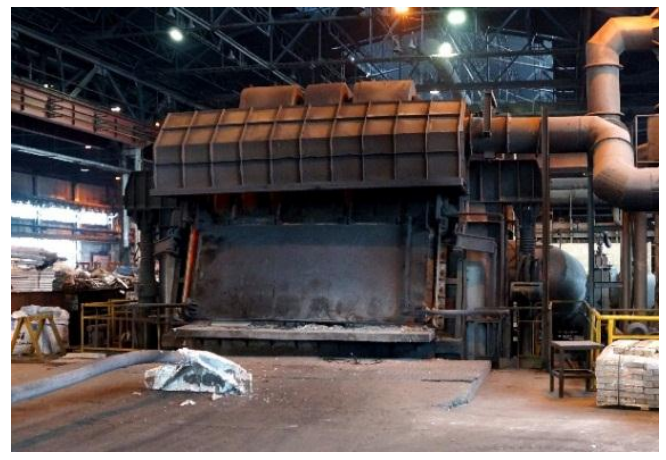

a

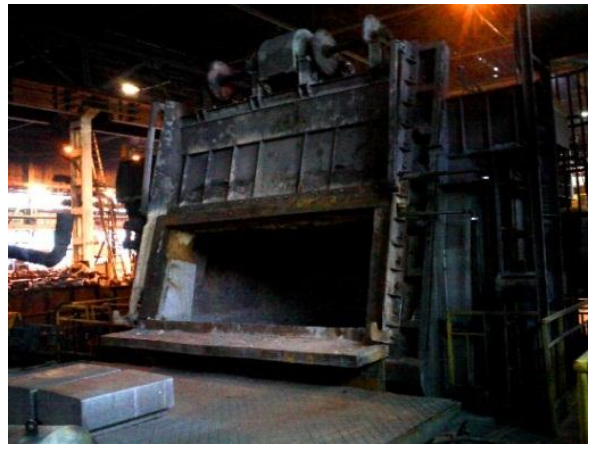

b

Fig. 1. General view of the furnace for melting aluminum.

In addition to transverse field inductors, longitudinal field LIM is used in electromagnetic stirrers of aluminum melt [9]. Specific modes of induction complexes lead to problems of electromagnetic compatibility (EC) of equipment and electrical networks [10 - 12]. In addition to the traditional issues of ensuring the quality of electricity, there are problems of controlling the configuration and intensity of the external electromagnetic field of inductors $[13,14]$. At the same time, linear induction machines with open magnetic circuits with a linear current load of up to $105 \mathrm{~A} / \mathrm{m}$ have significant dissipation, which is difficult to limit by electromagnetic shielding. When placing inductors under the bottom of the furnace, the most widespread are inductors of a transverse magnetic field, which ensure proper mixing efficiency of the melt at sufficiently large non-magnetic gaps $[15,16]$.

One of the varieties of such inductors can be considered flat toothless induction machines with an inclined arrangement of windings or stepped windings [1, 17]. The magnetic circuit of the toothless inductor is open, it is located outside the windings and acts as an electromagnetic shield. The inductors of linear MHD machines are usually calculated using engineering methods and then verified by mathematical modeling. The power supply system with different circuits for switching on coils and sections is investigated using circuit simulators. Diagrams for connecting induction complexes to a distribution network when designing an MHD mixer are considered simplified, without taking into account the location of distribution points and the features of cable connection. For EC reasons, it is preferable to 
use an isolation power transformer at the input of the frequency converter. This solution significantly reduces the effect of non-sinusoidal currents consumed by the inverter on the network mode.

The practical purpose of the study is to solve the problem of equipping the furnace with an induction complex. Mechanical stirring of the melt is of inadequate quality and is obsolete. The study should be comprehensive in order to propose options for improving the characteristics of the complex, taking into account electromagnetic compatibility. For this, in addition to traditional transverse field inductors, it is necessary to investigate the characteristics of a modified linear machine with a toothless design. In a field model, an external core inductor with improved shielding conditions should be evaluated. In addition to models for analyzing the electromagnetic field, it is necessary to propose models of power circuits that take into account the features of two-frequency and non-sinusoidal power modes.

\section{Methods}

The solution to the problem is performed using computational modeling. In the analysis of power circuits, the simulator software is used, designed for the analysis of induction devices $[4,18]$. The parameters of the elements and models are refined using the simulation of a stationary three-dimensional electromagnetic field by finite element methods in the Ansys software environment [19]. The induction machines used in the mixer can vary, depending on the conditions for ensuring the best EC, therefore, a complex of numerical models is being developed, ensuring their parametric properties. A sketch of the parametric model of the induction machine under the furnace and some of the results of the field simulation are shown in Fig. 2.

A schematic representation of the design of a flat toothless four-zone MHD inductor used in the complex for electromagnetic stirring of liquid aluminum is shown in Fig. 2. The features of the presented device include the splitting of the winding of the first phase (Fig. 2) and a special stepped shape of the inductive coils, which provides increased thrust, as well as the absence of saturated steel teeth, which helps to reduce losses.
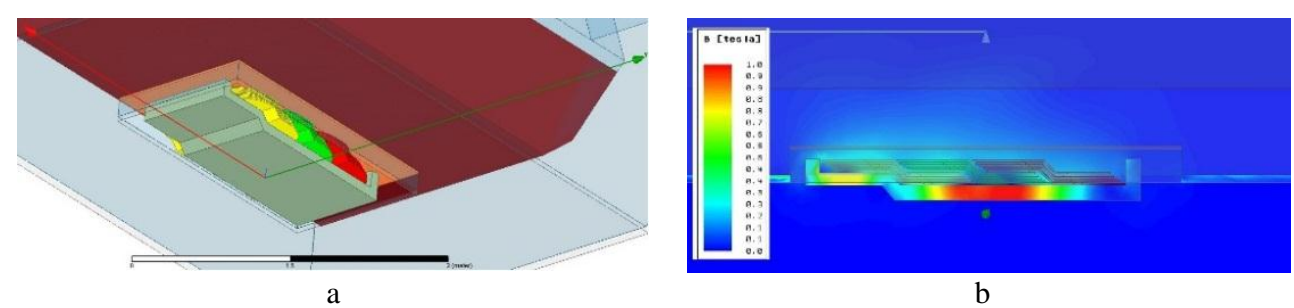

Fig. 2. Sketch of the model (a) in the Maxwell software environment and the calculation results (b).

The design of the inductor includes traction windings 1 of different phases and a laminated magnetic circuit 2, which acts as an electromagnetic shield (Fig. 3, a). The design of the winding sections (Fig. 3, b) is somewhat complicated in comparison with traditional disc double-layer coils. This solution is designed to correct the direction of the magnetic induction vector and change the direction of thrust. The stepped shape of the windings can be provided by pressing before the sections are impregnated with an organosilicon composition. Solidity and strength of the windings are given by baking in a technological oven [5]. 

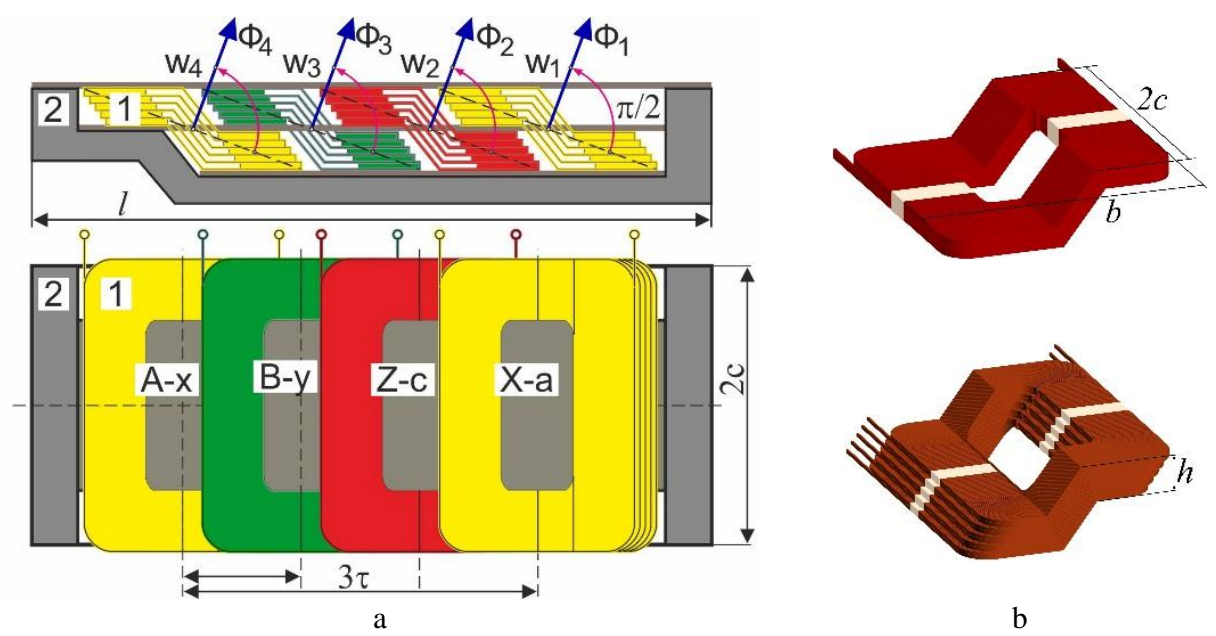

b

Fig. 3. Design and windings of the toothless four-zone inductor of the MHD-complex.

The windings of linear induction machines are quite diverse $[5,7,15,18]$. LIM inductors are made, two-phase, three-phase and multi-phase, and the number of windings can be odd or even. The windings themselves can be asymmetrical, and the magnetic core of the inductor is always open. Most often, three-phase and multi-phase windings are included according to the delta pattern.By changing the circuit of the windings, the modes of their power supply or the shape of the current, it is possible to gain advantages in creating traction forces in the melt. This helps to improve the efficiency of induction machines and improve the technology in general $[15,18]$. A similar means of influencing the redistribution of forces in the melt and the peculiarities of mixing can be considered a two-frequency power supply of the induction machine. By choosing the appropriate current frequencies during PWM modulation, the beat mode is reached with a significant increase in the voltage amplitude on the inductive windings.

The diagram for connecting the windings of a three-zone three-phase toothless inductor to the inverter is shown in Fig. 4. The traditional inclusion of the middle phase winding with inversion to obtain the AYC or AZB circuits will certainly be preserved. It can be noted that the cross-circuits indicated in the diagram are characteristic of the ideal case, which provides for a detailed account of the effect of the windings.

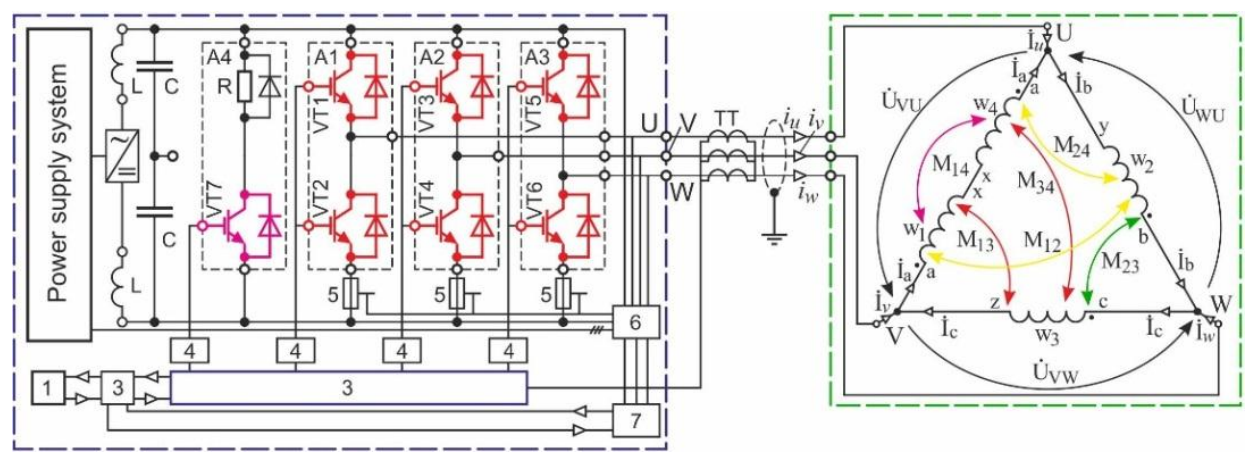

Fig. 4. Simplified diagram of connection of a toothless inductor to an inverter.

However, in practice, for the magnetic field of open magnetic circuits, in most cases, it is not required to take into account the influence on each other of the extreme windings located at a significant distance. Therefore, when modeling the power circuits of inductors, it is 
necessary to synthesize circuit models that are adequate to the conditions specified in the calculation.

The presented inverter connection diagram can be considered traditional, taking into account the fact that the converter is designed for use with asymmetric MHD machines and provides trouble-free operation of static induction load in the conditions of metallurgical production [20]. The structure and features of the modes of such a transistor inverter are described in the literature [21,22]. Adopted in Fig. 4 designations determine the purpose of the main elements: 1 - control panel, 2 - control module board, 3 - PWM controller, 4 - power module driver, 5 - power switch fuses, 6 - voltage converter, 7 - control system commutation board. The elements of the complex have a number of features, among which we can mention module 3, with elements of the dual-frequency power supply subsystem.

An example of a circuit model for switching on the windings of a three-phase MHD inductor when powered from an idealized two-frequency source is shown in Fig. 5. The analysis of the mode of the power supply system is carried out in the time domain, by analogy with the dynamics of the transient mode. Simulation is performed numerically using discretization algorithms for reactivity and nonlinearity models [23 - 25]. In the schematic model, enlarged, the lumped parameters of the source and connecting cables are taken into account.

To take into account the mutual inductance of neighboring windings, the model uses the technique of including a pair of controlled sources in the gap of the triangle connection. It is known that the EMF of mutual induction is determined by the derivative of the mutual current

$$
e_{\mathrm{i}}^{M}(t)=M_{(\mathrm{ij})} \frac{d i_{\mathrm{j}}(t)}{d t}, \quad \quad e_{\mathrm{j}}^{M}(t)=M_{(\mathrm{ji})} \frac{d i_{\mathrm{i}}(t)}{d t} .
$$

For the used voltage sources controlled by current (VSCC) in the circuit in Fig. 5, the introduced EMF of the opposite branch is characteristic, determined by the transfer coefficient $\pm \mathrm{k}_{\mathrm{M}}$

$$
e_{\mathrm{i}}^{M}(t)= \pm k_{M(\mathrm{ij})} \cdot i_{\mathrm{j}}(t), \quad e_{\mathrm{j}}^{M}(t)= \pm k_{M(\mathrm{ji})} \cdot i_{\mathrm{i}}(t) .
$$

Therefore, the value $\pm \mathrm{k}_{\mathrm{M}}$ can be represented as a set of discrete values of the matrix transfer coefficient

$$
\pm k_{M(\mathrm{ij})}=M_{(\mathrm{ij})} \frac{d i_{\mathrm{i}}(t)}{d t} / i_{\mathrm{i}}(t), \quad \quad \pm k_{M(\mathrm{ji})}=M_{(\mathrm{ji})} \frac{d i_{\mathrm{j}}(t)}{d t} / i_{\mathrm{j}}(t) .
$$

In the general case, under the conditions of discretization of reactivities used in most simulators, the matrix of coefficients are not equal and not reciprocal [26, 27]. However, in practice, when calculating devices with an open magnetic circuit, the differences in the values of the coefficients on different sides of the main diagonal can be neglected, while maintaining acceptable accuracy.

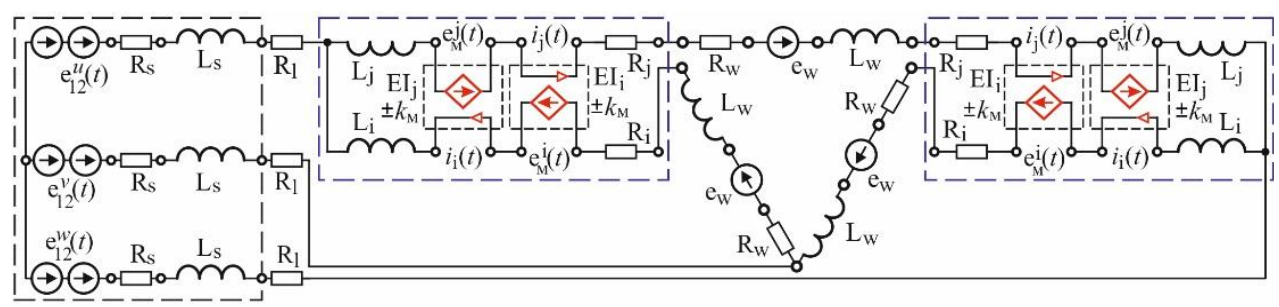

Fig. 5. Schematic model of the MHD-complex when connecting the windings in a triangle.

This greatly simplifies the circuitry of models with inductive coupling. The values of the transfer coefficient $\pm \mathrm{k}_{\mathrm{M}}$ of each controlled source are set in a table, in accordance with the current values of the operating parameters determined at each step of the iterative calculation $[24,27,28]$. The deliberate simplification of the circuitry is based on the fact that inductive 
couplings $\mathrm{M}_{12}$ and $\mathrm{M}_{34}$ of the pairs of extreme windings $\mathrm{w}_{1}-\mathrm{w}_{2}$ and $\mathrm{w}_{3}-\mathrm{w}_{4}$ are essential. Since the cores of the inductors of linear machines are open, the inductive coupling of the extreme windings $\mathrm{w}_{1}-\mathrm{w}_{4}$ with each other can be considered insignificant and not taken into account $\left(\mathrm{M}_{14}\right)$ in the simulation.

Calculations have shown that an increase in the coefficient of current asymmetry in a triangle by $1-2 \%$ for detailed calculations of the mode of an inductor with a sinusoidal source, in comparison with the resulting asymmetry of the linear currents of the inverter in $25-35 \%$, is not decisive. For the case of using a two-frequency device in the power supply system, it is possible to show the calculated ratios characteristic of the addition of the harmonic functions of any phase in relation to a three-phase source:

$$
\begin{aligned}
& u(t)=u_{0}\left(\cos \omega_{1} t+\cos \omega_{2} t\right)=2 u_{0} \cos \left[\left(\omega_{1}-\omega_{2}\right) \cdot t / 2\right] \cdot \cos \left[\left(\omega_{1}+\omega_{2}\right) \cdot t / 2\right] \\
& u(t)=2 u_{0} \cos \left[\omega_{12}^{-} \cdot t\right] \cdot \cos \left[\omega_{12}^{+} \cdot t\right]=U(t) \cos \left[\omega_{12}^{+} \cdot t\right], \quad \omega_{12}^{+}=\left(\omega_{1}+\omega_{2}\right) / 2 .
\end{aligned}
$$

where the amplitude of the new harmonic oscillation changes slowly and is denoted by $U(t)$

$$
U(t)=2 u_{0} \cos \left[\omega_{12}^{-} \cdot t\right], \omega_{12}^{-}=\left(\omega_{1}-\omega_{2}\right) / 2 .
$$

The result obtained is usually interpreted as a dynamic amplitude. Therefore, in the graphs when simulating a two-frequency power supply of an MHD inductor, it can be seen that for two voltage sources with a frequency of 1.0 and $1.25 \mathrm{~Hz}$, the frequency difference is 0.25 $\mathrm{Hz}$. This means that the envelope period is $\mathrm{T}=4 \mathrm{~s}$.

\section{Results}

Characteristics of instantaneous voltages in the phases of a dual three-phase source are shown in Fig. 6. Special symbols mark the curve for the first phase alternation in direct sequence. The simplest solution here is to replace the module of an idealized power supply with EMF sources $\left(\mathrm{e}_{\mathrm{u}}(\mathrm{t}), \mathrm{e}_{\mathrm{v}}(\mathrm{t}), \mathrm{e}_{\mathrm{w}}(\mathrm{t})\right.$ ), with a dual one (index - 12), built on the basis of series connection of sinusoidal EMF sources (Fig. 5) or parallel connection current sources $\left(\mathrm{j}_{\mathrm{u}}(\mathrm{t})\right.$, $\left.\mathrm{j}_{\mathrm{v}}(\mathrm{t}), \mathrm{j}_{\mathrm{w}}(\mathrm{t})\right)$.

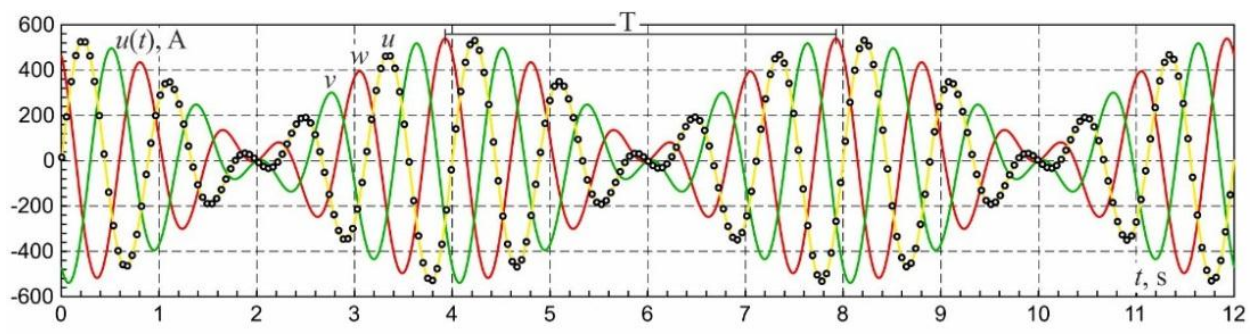

Fig. 6. Curves of instantaneous voltage of LIM windings with dual-frequency power supply.

The results of modeling instantaneous current curves in the inductor windings with allowance for inductive coupling are shown in Fig. 7. A significant violation of the symmetry of the currents in the graphs is obvious (double amplitudes are highlighted). However, it is premature to speak of non-sinusoidality in the investigated mode, since the model did not use the saturation characteristics of the steel core.

It should be noted that for the proposed toothless inductor, the saturation onset boundaries are located farther in comparison with traditional transverse field inductors [1, 7]. And even further, in comparison with longitudinal field inductors, in which the windings cover the yoke [9]. Therefore, the real values of the relative magnetic permeability $\mu=\mathrm{f}(B)$ in the shielding magnetic circuit, as well as the degree of saturation of steel, can be obtained by comparative mathematical modeling of the electromagnetic field, observing the similarity of modes. 


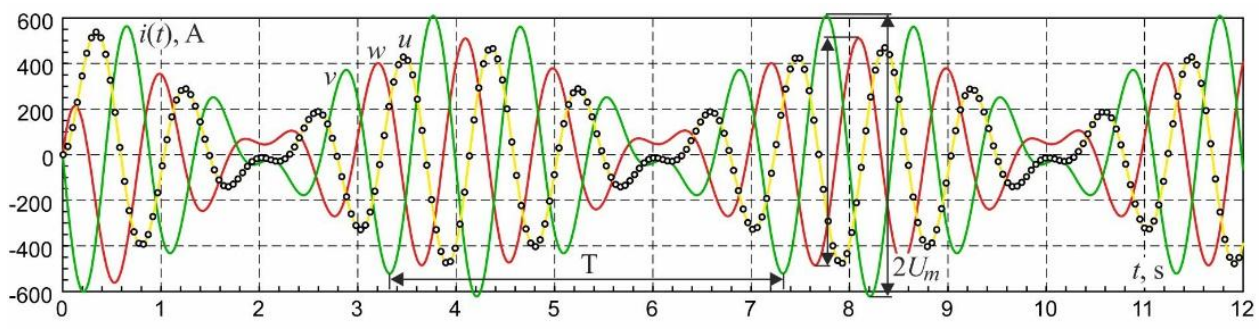

Fig. 7. Curves of instantaneous current of windings with dual-frequency power supply.

To test the signals of arbitrary complexity modulating the power supply system, a circuit model is synthesized from the standard elements of the simulator. Examples of typical superposition schemes, where sources of EMF and current are used to create a signal, are shown in Fig. 8, a, b.
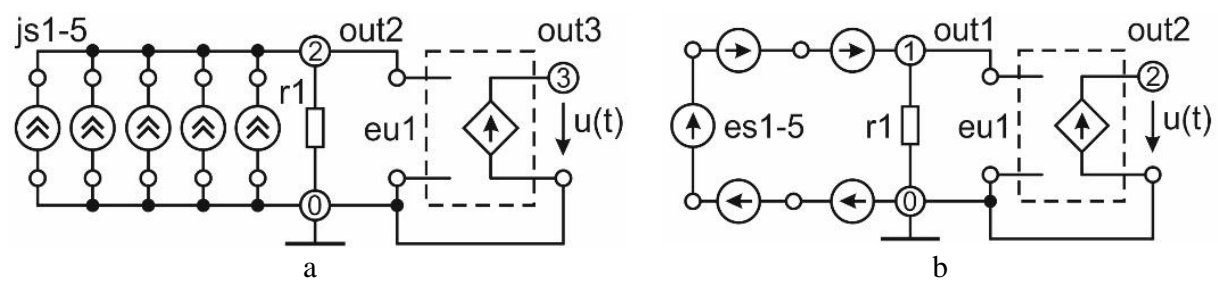

Fig. 8. Schematic models of sources.

For an arbitrary number of current harmonics, use the circuit in Fig. 8, a. When using idealized EMF sources, use the circuit in Fig. 8, b. For galvanic isolation from the macromodel, a voltage controlled voltage source (VSVC) is used. When constructing a beat model, pairs of sources with close frequencies are used [29]. 1 .

Description of the simplified model with current sources (Fig. 8, a) is presented in table.

Table 1. Description of the circuit model.

\begin{tabular}{|c|c|c|c|}
\hline js $1\left(\begin{array}{ll}(0 & 2\end{array}\right) 122180$ & js $2\left(\begin{array}{ll}0 & 2\end{array}\right) 1200$ & \begin{tabular}{|lllll} 
eu $1\left(\begin{array}{llll}2 & 0 & 0 & 3\end{array}\right)$ & 1
\end{tabular} & $\mathrm{r} 1\left(\begin{array}{ll}2 & 0\end{array}\right) 1$ \\
\hline
\end{tabular}

For two low frequency harmonics, beats were obtained, the parameters of which can be determined from the characteristics of the current sources.

$$
j_{1}(t)=J_{m 1} \sin \left(\omega_{1} t+\psi_{1}\right), \quad j_{2}(t)=J_{m 2} \sin \left(\omega_{2} t+\psi_{2}\right),
$$

where $\mathrm{Jm} 1=\mathrm{Jm} 2=\mathrm{Jm}=1 \mathrm{~A}, \mathrm{fl}=22 \mathrm{~Hz}, \mathrm{f} 2=20 \mathrm{~Hz}, \omega=2 \pi \mathrm{f}, \psi 1=\pi, \psi 2=0$.

The resulting signal at the output of the source model is determined by the expression:

$j(t)=j_{1}(t)+j_{2}(t)=J_{m}\left[\sin \left(\omega_{1} t+\psi_{1}\right)+\sin \omega_{2} t\right]$.

After completing the trigonometric transformation, write down the formula:

$j(t)=J_{m}\left\{\sin \left[\left(\omega_{1}+\omega_{2}\right) /(2 t)+\pi / 2\right]+\cos \left[\left(\omega_{1}-\omega_{2}\right) /(2 t)+\pi / 2\right]\right\}$.

An example of modeling the characteristics of a modulated voltage source in the time domain is shown in Fig. 9. 


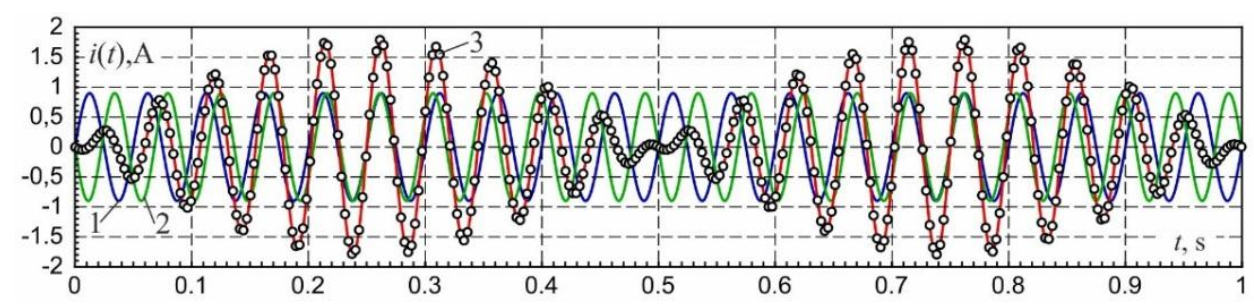

Fig. 9. Curves of the beating source in the time domain.

By substituting the frequency expressions, we get:

$$
j(t)=J_{\mathrm{ml}} \sin (\omega \mathrm{t}+\pi / 2)+J_{\mathrm{m} 2} \cos (\Omega \mathrm{t}+\pi / 2),
$$

Where $\omega=\left(\omega_{1}+\omega_{2}\right) / 2=(44 \pi+40 \pi) / 2=42 \pi \mathrm{s}^{-1}$,

$$
\Omega=\left(\omega_{1}-\omega_{2}\right) / 2=(44 \pi-40 \pi) / 2=2 \pi \mathrm{s}^{-1} .
$$

In this case, the value of the beat period is determined by the formula:

$\mathrm{T}=\pi / \Omega=1 / 2=0.5 s$.

In addition to simply converting the characteristics of two sinusoidal signals equivalently, modulation capabilities can be used to obtain more complex characteristics of currents and voltages in the power supply system. Such an approach can make it possible to change the nature of the pulse power supply of the windings of induction machines. It is one thing for a three-phase inverter to generate periodic currents with a frequency of 1-5 Hz, a complex shape, with a large steepness of the fronts, for example, trapezoidal ones.

Obtaining such currents in the windings of a magnetically coupled inductor is not an easy task. It may be somewhat simpler to construct periodic non-sinusoidal currents as a result of envelope extraction from an amplitude-modulated harmonic of 100-200 Hz. Moreover, the result of the transient process, in relation to overvoltages, taking into account the PWM source (1-2 kHz), for periodic currents synthesized from harmonics, may turn out to be much softer than from the trapezoid. At the stage of modeling by means of the elemental basis of the simulator, it is possible to perform the procedures for constructing rather complex sources of the control signal $[3,4]$. Examples of applying amplitude, phase and frequency modulation of an arbitrary sinusoidal source are shown below. The modes of programmable sinusoidal sources of EMF Es and current Js have been implemented.

The voltage characteristics of the model with amplitude modulation (AM) in the time domain are shown in Fig. 10. The parameters of the modulating effect of a trapezoidal pulse on the harmonic with a frequency of $f=500 \mathrm{~Hz}$ are given in a table (Table 2) for the Es 1 element. With amplitude modulation, the width of the frequency spectrum of the modulated signal does not depend on the intensity of the modulating signal. The description of the model with amplitude-phase modulation in the standard shell of the language basis of the simulator is shown in Table. 2. Tabular setting of the modulating amplitude is based on the coordinates of 6 points (npunct $=6$ ).

In the general case, each active or passive element of the standard library can have a matrix description in accordance with the features of its nonlinearity or the parametric nature of setting properties in the time domain. In this case, the iteration step is determined automatically, and, if necessary, control of the computation step is provided. In the frequency domain, the macro model is linearized according to the indication of the control subroutine.

\begin{tabular}{|c|c|c|c|c|}
\hline r 1 & out $1\left(\begin{array}{ll}1 & 0\end{array}\right)$ & es 1 tab $(01) 15000$ & npunct $=6$ & \\
\hline 0,00 & 0,005 & 0,01511 & $\longdiv { 1 1 }$ & 011 \\
\hline
\end{tabular}

Table 2. Description of the circuit model. 
An example of the voltage characteristics of the source model in the time domain in the presence of amplitude (AM) and phase modulation (PM) is shown in Fig. 10.

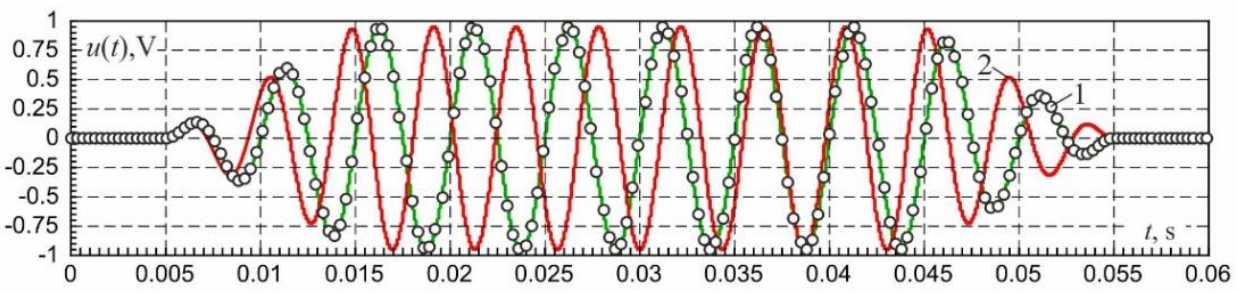

Fig. 10. Characteristics of $A M$ and $P M$ modulated voltage.

The description of the model when programming amplitude and phase modulation, providing a phase shift of $3 / 2$ period $\alpha=540 \mathrm{deg}$.) Is given in table. 3 . With phase modulation, an increment proportional to $\mathrm{x}(\mathrm{t})$ is obtained by the phase of the carrier waves. $\varphi_{\mathrm{H}}=\varphi 0+\Delta \varphi \mathrm{x}(\mathrm{t})$. The quantity $\Delta \varphi$ is called phase deviation and defines the concept of modulation depth.

Table 3. Description of the circuit model.

\begin{tabular}{|c|c|c|c|c|c|}
\hline $\mathrm{r} 11$ & out $1\left(\begin{array}{ll}1 & 0\end{array}\right)$ & es 1 tab $\left(\begin{array}{lll}0 & 1\end{array}\right)$ & 2001 & $=6$ & \\
\hline 0,00 & $0,005011 \mathrm{e}-6$ & 0,01511108 & 0,04511432 & 0,05501540 & 0,0601540 \\
\hline
\end{tabular}

The simulator provides the ability to build a model of a pulse source with amplitudefrequency modulation [30]. For the required regularities of the simultaneous application to the standard sinusoid of all possible types of modulation, tabular programming of characteristics can be applied.

An example of instantaneous voltage curves of a source model for combined application of amplitude and linear frequency modulation (FM) of a sinusoidal signal is shown in Fig. 11.

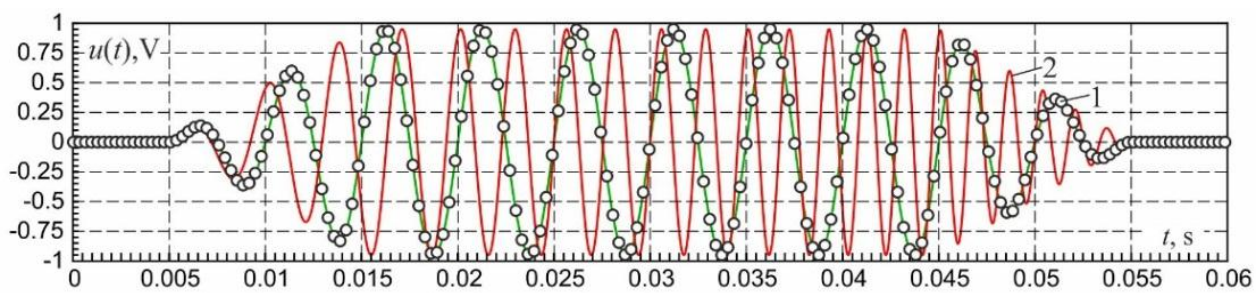

Fig. 11. Characteristics of AM and FM modulated voltage.

The parameters of the frequency modulating (FM) influence on the harmonic of a sinusoid with a frequency of $\mathrm{f}=200 \mathrm{~Hz}$ for Es1 are given in a table (Table 4) by six points. With frequency modulation, an increment proportional to the value of $\mathrm{x}(\mathrm{t})$ receives the carrier frequency $\omega_{\mathrm{H}}=\omega 0+\Delta \omega \mathrm{x}(\mathrm{t})$. The value of $\Delta \omega$ is called the frequency deviation, it determines the depth of modulation.

Table 4. Description of the circuit model.

\begin{tabular}{|c|c|c|c|c|c|}
\hline r $1(10) 10,0$ & out $1\left(\begin{array}{ll}1 & 0\end{array}\right)$ & es 1 tab $\left(\begin{array}{ll}0 & 1\end{array}\right)$ & 2000 & npunct $=6$ & \\
\hline 0,0000 & 0,005011 & \begin{tabular}{|lllll}
0,015 & 1 & 1,2 & 1
\end{tabular} & $0,04511,81$ & $0,05502,01$ & 2,11 \\
\hline
\end{tabular}

It can be concluded that phase and frequency modulation are similar phenomena, since PM can be considered as a kind of frequency modulation. The width of the frequency modulation spectrum can be determined by the expression $\omega_{C}=2 \Delta \omega$. This value is called the swing band, since in the process of modulation according to an arbitrary given law, the 
frequency can take any instantaneous value in the range of frequencies under consideration: $\omega=\omega 0 \pm \Delta \omega$. It is important to note that in FM modulation, the width of the discrete frequency spectrum is proportional to the amplitude of the applied modulating signal.

An example of a study in a simulator of a three-phase model of a non-sinusoidal source according to Fig. $7 \mathrm{~b}$ is discussed below. The characteristics of rectangular pulses are shown in Fig. 12 in two versions.

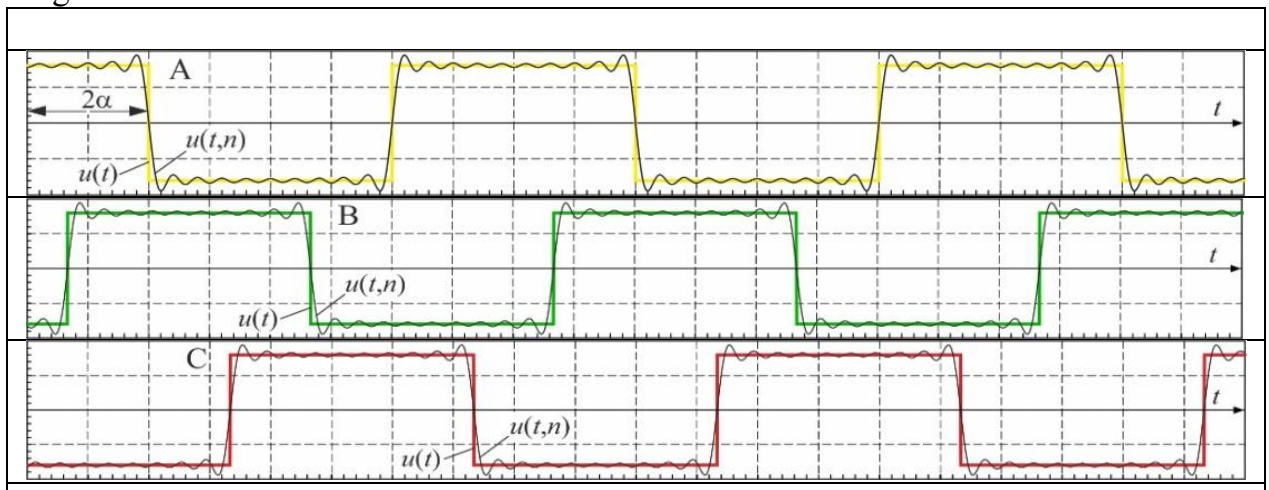

Fig. 12. Characteristics of the model of a three-phase non-sinusoidal voltage source.

The representation of the model by a set of sinusoidal current sources (Fig. 8, a) corresponds to the approximate monochrome curves $u(t, n)$, synthesized from $n=20$ harmonics. The impulse representation of voltages in phases is characterized by ideal rectangular curves $\mathrm{u}(\mathrm{t})$ highlighted in color. For representation in the time domain, the most significant harmonics are highlighted in color, with numbers 1, 3, 5. Using three-phase models and characteristics, a multivariate study of an induction device in a steady-state nonsinusoidal mode is performed, revealing the features of the asymmetry of power circuits, taking into account the inductive connections between the windings for a triangle connection $[17,20]$. In addition to statics, the behavior of the system in dynamics is investigated, evaluating the correctness of the decisions made for a source synthesized using the superposition principle and models of mutual inductances of adjacent phases.

A typical set of sinusoidal curves indicating the numbers of harmonics in phase A of a three-phase source, obtained by modeling during spectral analysis for Fig. 12 is shown in Fig. 13.

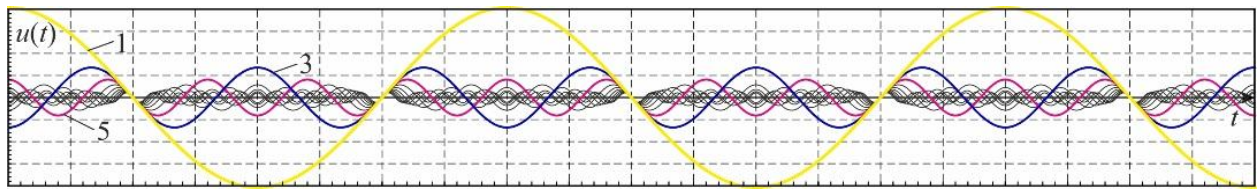

Fig. 13. Spectral composition of voltage harmonics of phase A.

When calculating the modes of power circuits in the Mathcad environment, an analytical expression synthesized for the trigonometric Fourier series can be used. The formula describes the sequence of pulses in phase $\mathrm{A}$ and has the following form:

$$
u(\omega t)=\frac{2 U_{m}}{\pi}\left\{\sum_{k=1}^{n} \frac{\left[(-1)^{k+1}+1\right] \sin (k \alpha)}{k} \cos (k \omega t)+\sum_{k=1}^{n} \frac{\left[(-1)^{k}-1\right] \cos (k \alpha)}{k} \sin (k \omega t)\right\},
$$

where $\alpha=\pi / 4$ is the phase coefficient.

\section{Discussion}


When using the hardware of the simulator, it is possible to implement experimental bench devices equipped with universal measuring instruments built on the basis of a library of virtual prototypes. Even more diverse signal characteristics can be learned by using programmable models of switching sources, which are then used to simulate non-sinusoidal modes of power supply systems for multiphase induction devices $[6,16]$. To study the features of the pulsed power supply of the windings of MHD inductors, it is necessary to synthesize the corresponding three-phase or multiphase models of pulsed current sources.

A practical assessment of the prospects for power supply of the windings of a multiphase inductor with non-sinusoidal currents will require additional research. At the same time, it is initially obvious that the power supply of the mixer from sources of single current pulses is ineffective and impractical due to the low power density. The use of special shaped pulse trains for power supply of inductors, especially at low frequencies, is quite possible. However, the practical implementation of this approach, in view of the probability of overvoltage, will require a preliminary calculation and careful modeling [18, 25]. Since the voltage across the inductor (as well as in the source) is proportional to the derivative of the current, a sequence of current pulses with a large steep front can lead to periodic overvoltages, along a curve, with practically unlimited amplitude. Therefore, special protective measures and appropriate circuit solutions are needed when building the power link of an IGBT inverter.

In addition to the above, the use of periodic non-sinusoidal currents in inductors will require a separate careful assessment of power consumption modes from the standpoint of electromagnetic compatibility of induction equipment with a distribution network [31]. Indeed, even with sinusoidal inductor currents of 300-400 amperes, the distortion factor of currents consumed from the network can reach $50-60 \%$, in the presence of complex modulation with a frequency of $1-2 \mathrm{~Hz}$.

\section{Conclusion}

The article discusses models of two-frequency and combined power supply of the MHD inductor windings of a metallurgical linear machine. The advantage of the dual-frequency power supply can be considered a decrease in the loads on the power modules of the transistor frequency converter. The outlined approach determines the construction of either a dual power link of the inverter, or the corresponding complication of the control system. A significant consequence of the two-frequency approach is a change in the power supply mode of the LIM windings, a change in the frequency of the current. To study dual-frequency and non-sinusoidal modes, it is required to build sophisticated models and refine the modeling technique. The technological features of the use of two-frequency and non-sinusoidal currents to increase the traction efficiency of inductors with different combinations of windings require a separate study.

The use of pulsed currents for powering the windings of an induction machine should be treated with some caution, since the values of the LIM's own inductances are very significant. Powerful inductors are characterized by an extremely low value of the natural power factor, which means significant asymmetrical reactive currents with equivalent losses of the complex up to $100 \mathrm{~kW}$. Even if modules with a maximum permissible voltage of $4-5 \mathrm{kV}$ are used in transistor inverters, the dynamic overvoltage due to the high steepness of the current pulse can significantly exceed the design limits. Therefore, it is preferable to solve the problem of increasing the stirring efficiency by using non-sinusoidal currents in LIM windings at the stage of mathematical modeling. At the same time, a physical experiment is of decisive importance in the implementation of this approach. 


\section{References}

1. A. Tyapin, E. Kinev, The scientific heritage, 55-1 (1), 67 (2020)

2. A.A. Tyapin, E.S. Kinev, The scientific heritage, 51-1(1), 63 (2020)

3. A.A. Tyapin, E.S. Kinev, Materials of the II All-Russian Scientific and Practical Conference, 2, 135 (2020)

4. A.A. Tyapin, E.S. Kinev, III International Scientific and Practical Conference, 96 (2019)

5. S.A. Bychkov, Winding of induction machines of rotational and translational motion (Yekaterinburg: UrFU, 2017)

6. V.N. Timofeev, G.F. Lybzikov, M.Yu. Khatsyuk, M.A. Eremin, S.P. Timofeev, Journal of the Siberian Federal University. Series: Engineering and technology, 6 (2), 166 (2013)

7. Patent RU 2683596 C1. Timofeev V.N. Inductor of a linear induction machine. Application: 2018117423. Published on March 29, 2019.

8. A.A. Maksimov, M.Yu. Khatsyuk, V.N. Timofeev, Light alloy technology, 4, 106 (2018)

9. Patent RU 2708036 C1. Golovenko E.A., Avdulov A.A., Kinev E.S., Timoshev V.E. A method for stirring a metal melt and an electromagnetic stirrer for its implementation. Registered 05.10. Published: 03.12.2019.

10. G.N. Chistyakov, E.V. Platonova, T.Yu. Zarubina, V.I. Panteleev, Electromagnetic compatibility technologies, 2(61), 25 (2017)

11. E. Khabiger, Energoatomizdat (1995)

12. L.E. Roginskaya, E.N. Gulyaev, Bulletin of the Chuvash University, 3, 244 (2010)

13. A.L. Badalov, A.S. Mikhailov, Norms for parameters of electromagnetic compatibility of radio-electronic means: Handbook (M.: Radio and communication, 1990)

14. V.M. Saltykov, V.M. Barinov, Electromagnetic compatibility technologies, 2(69), 73 (2019)

15. Patent RU 2524463 C2. Timofeev V.N., Lybzikov G.F., Khatsyuk M.Yu., Eremin M.A. Induction plant for stirring liquid metals, published on 07/27/2014. Application No. 2012146779/02 dated 01.11.2012.

16. Patent RU 2680715 C1. Lybzikov G.F., Timofeev V.N. Power supply for the inductor. Published on February 26th, 2019. Application No. 2017138175 dated 01.11.2017.

17. A.A. Tyapin, E.S. Kinev, Collection of scientific works of the 67th International Scientific Conference of the Eurasian Scientific Association, 9(67-2), 145 (2020)

18. E.S. Kinev, A.A. Tyapin, Collection of scientific works of the 65th International Scientific Conference of the Eurasian Scientific Association, 7(65-2), 110 (2020)

19. ANSYS 2019 R1. Updates and Changes (2019)

20. E. Kinev, A. Tyapin, Sciences of Europe, 52-2, 61 (2020)

21. B.W. Williams, Principles and Elements of Power Electronics. Devices, Drivers, Applications and Passive Components (Glasgow, United Kingdom, 2006)

22. The Industrial Electronics Handbook. Power electronics and motor drives (Taylor and Francis Group, LLC, Boca Raton, London, New York, USA, 2011)

23. L.O. Chua, Computer analysis of electronic circuits: Algorithms and computational methods (Moscow: Energiya Publishing house, 1980)

24. L.O. Chua, C. Desoer, E. Kuh, Linear and nonlinear circuits (McGraw-Hill, New York, 1987) 
25. R. Shaffer, Fundamentals of Power Electronics with MATLAB (Charles River Media, Boston, Massachusetts, USA, 2007)

26. G.V. Zeveke P.A. Ionkin, A.V. Netushil, S.V. Strakhov, Fundamentals of circuit theory (M.: Energoatomizdat Publishing house, 1989)

27. E.A. Karpov, V.N. Timofeev, Yu.S. Perfilyev, M.Yu. Khatsyuk, M.V. Pervukhin, Modeling of transient processes in linear and non-linear electrical circuits (Krasnoyarsk: Siberian Federal University, 2019)

28. E. Kinev, A. Tyapin, V. Panteleev, M. Pervukhin, The scientific heritage, 63-1(1), 53 (2021)

29. Yu.A. Bychkov, V.M. Zolotnitsky, E.P. Chernyshev, A.N. Belyanin, Fundamentals of theoretical electrical engineering (Lan" Publishing house, 2008)

30. S.I. Baskakov, Radio circuits and signals (Moscow: "Higher school" Publishing house, 2005)

31. K.G. Gainullin, A.N. Getmanets, A.N. Grebennikov, D.A. Kaidarov, E.A. Sarycheva Electromagnetic compatibility technologies, 4(71), 16 (2019) 